\title{
Proposta de controlador não linear para atitude de nanossatélite baseada em cinemática descrita por quatérnios
}

\author{
Samaherni M. Dias* Kurios I. P. M. Queiroz* \\ Aldayr D. Araújo* \\ * Laboratório de Automação, Controle e Instrumentação (LACI), \\ UFRN, Natal, RN, Brasil, (sama@laci.ufrn.br, kurios@laci.ufrn.br, \\ aldayr@dca.ufrn.br)
}

\begin{abstract}
Interest in the CubeSat class of nanosatellites has surged in recent years. In Brazil, some projects based on CubeSat class have been developed, and one of these projects in development is the Nanosatellite Constellation for Environmental Data Collection (CONASAT), which is developed by the INPE/CRN and UFRN. The main objective of this work is to modify the attitude control law of the nanosatellites (that uses magnetorquers as the only active torque source) to reduces the power consumption during the stabilization process. In this work is proposed adding a nonlinear function to compensate for the coupling effects that exists in the traditional attitude control. Some simulation results are shown to exhibit the performance of the proposed controller.

Resumo: O interesse na classe CubeSat de nanossatélites aumentou nos últimos anos. No Brasil, alguns projetos com base na plataforma de CubeSat têm surgido, e dentre eles está o projeto CONASAT, o qual é desenvolvido pelo INPE/CRN e UFRN, que tem o objetivo de desenvolver uma constelação de nanossatélites para coleta de dados ambientais. Este trabalho apresenta alterações no controle de atitude do nanossatélite (atuado através de bobinas magnéticas) que está sendo desenvolvido no projeto CONASAT, com o objetivo de reduzir o consumo de energia utilizado na estabilização do satélite em órbita. Para isto, será adicionada à lei de controle tradicional (PD), uma correção não linear visando compensar os efeitos dos acoplamentos existentes ao se acionar as bobinas magnéticas do nanossatélite. Resultados de simulação são apresentados para comprovar o funcionamento da técnica proposta.
\end{abstract}

Keywords: Theory of Control, Attitude Control, Nanossatellite, Quaterniums.

Palavras-chaves: Teoria de Controle, Controle de Atitude, Nanossatélite, Quatérnios.

\section{INTRODUÇÃO}

A tecnologia Cubesat oferece um caminho excepcionalmente barato para o estudo de fenômenos científicos e o avanço de novos conceitos de engenharia. Custos reduzidos para participar de atividades espaciais estimularam pequenos programas de desenvolvimento de satélites em todo o mundo pelos governos, indústria e, particularmente, instituições acadêmicas de um número crescente de nações com capacidades tecnológicas avançadas e emergentes, incluindo Argélia, Argentina, Brasil, Colômbia, Egito, Indonésia, Irã, Israel, Malásia, México, Nigéria, Paquistão, África do Sul, Coreia do Sul, Turquia e Venezuela (Woellert et al., 2011).

No Brasil, alguns projetos com base na plataforma $\mathrm{Cu}-$ beSat têm surgido nos últimos anos, como o NanosatCBr1, que tornou-se o primeiro CubeSat brasileiro, lançado em junho de 2014. Atualmente, o Centro Regional do Nordeste (CRN) do Instituto Nacional de Pesquisas Espaciais (INPE) está desenvolvendo a missão CONASAT, uma constelação de nanossatélites para coleta de dados ambientais, em cooperação com a Universidade Federal do Rio Grande do Norte (UFRN) e com apoio financeiro da Agência Espacial Brasileira (AEB). O CONASAT pretende estender o sistema brasileiro de coleta de dados ambientais, substituindo os satélites SCD-1 e SCD-2 por uma constelação de nanossatélites equipados com sistemas de comunicação digitais (Reis de Mesquita et al., 2017).

O satélite considerado neste trabalho será lançado em órbita baixa (LEO - Low Earth Orbit) seguindo as especificações do CubeSat com configuração em uma estrutura $1 \mathrm{U}$ (ver Figura 1), com um processador ARM9 como computador de bordo (OBC - On Board Computer), com seis sensores de sol analógicos, um em cada face do módulo, um magnetômetro de três eixos, um giroscópio MEMS que irá fornecer as medições das velocidades angulares nos três eixos e um conjunto de três bobinas magnéticas (magnetorques) para atuar sobre o movimento do corpo do satélite.

O CubeSat $1 \mathrm{U}$ tem uma especificação dada por um cubo de $10 \mathrm{~cm}$ de aresta, massa não superior a $1 \mathrm{~kg}$ e foi introduzida por Jordi Puig-Suari da California Polytechnic Institute e 


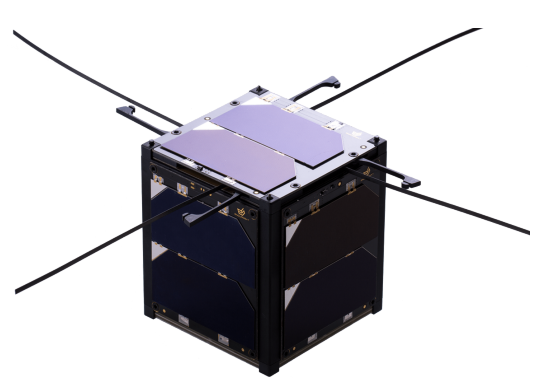

Figura 1. Estrutura CubeSat 1U

Fonte: www.endurosat.com

Robert Twiggs da University of Stanford. Hoje em dia, esta especificação ${ }^{1}$ é mais ampla ( $2 \mathrm{U}, 3 \mathrm{U}$ e etc) podendo envolver o agrupamento de mais de um cubo e massa superior a $1 \mathrm{~kg}$. Segundo Shiroma et al. (2011), os satélites podem ser classificados de acordo com sua massa. Neste trabalho, a classificação utilizada foi nanossatélite por se considerar um satélite com massa inferior a $10 \mathrm{~kg}$.

O controle da atitude de satélites é uma das questões mais importantes nas missões espaciais. Atualmente, para atender os requisitos destas missões, o projeto de controladores de atitude com alta precisão tornou-se cada vez mais importante. Apesar destes novos requisitos, o controlador PID (Proportional-Integral-Derivative) é o algoritmo de controle mais amplamente utilizado no controle da atitude de satélites (Li and Ye, 2019). Embora o PID ainda domine o cenário, várias abordagens por controladores não lineares, tais como controlador $\mathrm{H}_{\infty}$ (Show et al., 2003), controle adaptativo (Leeghim et al., 2009), controle backstepping (Kristiansen et al., 2009; Giri and Sinha, 2019), sliding mode control (Bang et al., 2005) e controladores ótimos (Lai et al., 2007), têm sido utilizados no problema do controle de atitude (Li et al., 2012).

O controle de atitude a partir da ação magnética (Wisniewski, 2000; Lovera et al., 2002; Ivanov et al., 2018; Giri and Sinha, 2019) é o mais comum para micro e nanossatélites em órbita baixa. Ele é usado para o amortecimento da velocidade angular, estabilização ao longo do vetor de indução geomagnética e estabilização do spin. A estabilização adequada requer a determinação em tempo real do movimento da atitude, o qual é obtido pelo processamento das medidas dos sensores de sol, magnetômetros, sensores de velocidade angular e até micro sensores de estrela. No entanto, a tendência para miniaturização e simplificação conduz os requisitos dos sistemas de controle a um conjunto mínimo de hardware (Ivanov et al., 2018).

Para atender os requisitos apresentados, proposições para o controle da atitude de nanossatélite devem ser ponderadas pela alocação de recursos do sistema de controle versus o desempenho. Desta forma, com o avanço da tecnologia dos sistemas embarcados, hoje em dia é possível se embarcar técnicas mais sofisticadas de controle em processadores de preço mais acessível. Assim, o custo para adequar o ADCS (Attitude-Determination-and-Control System, em português, sistema de controle e determinação de atitude) às necessidades de um sistema com configuração de hardware mais simples, muitas vezes é inferior ao custo do lançamento de um hardware mais sofisticado.

1 http://www.cubesat.org
O conceito de controle de atitude através da ação magnética considera a existência de uma única fonte de torque com atuação controlada, gerada pelos magnetorques, sobre o corpo do satélite. A interação entre os campos magnéticos da terra e os produzidos pelos magnetorques produzem o torque mecânico, sobre o corpo do satélite, o qual é utilizado para corrigir a atitude. O controle de atitude através da ação magnética, comparado a outras tecnologias empregadas em satélites, possui menor massa, requer menor potência e possui custo reduzido (Wisniewski, 2000).

Por fim, para os nanossatélites (1U), existe mais uma dificuldade, o sistema é alimentado por baterias que são carregadas por painéis solares sobre a superfície do cubo de aresta de $10 \mathrm{~cm}$. Como os satélites passam por zonas de sombra, faz-se necessária uma escolha adequada do sistema de energia (baterias e conversor). A utilização de um grande conjunto de baterias é inviável devido as restrições de tamanho e massa. Conversores de grande potência para uma rápida carga, também enfrentam problemas de tamanho e massa, além de gerar calor internamente. A solução, que não é tão simples, passa por sistemas embarcados mais eficientes. O ADCS compõe o sistema embarcado do satélite e pode contribuir na redução do consumo da energia das baterias adotando uma estratégia de controle de atitude mais eficiente energeticamente.

Este trabalho propõe a inserção de uma correção não linear ao controlador de atitude existente, baseado na aplicação de uma técnica de sistema inverso, com o objetivo de reduzir o consumo de energia das baterias, em contrapartida adicionando um pouco mais de complexidade à lei de controle. Esse incremento na complexidade da lei de controle é mais eficiente energicamente que aplicar o sinal de controle sem a correção.

\section{MODELO DO SATÉLITE}

O satélite neste trabalho é considerado um corpo rígido, somente atuado por ação magnética no qual o efeito da massa é incluído na matriz de inércia do satélite. Assumindo que existem dois referenciais pertencentes ao $\Re^{3}$ para descrever a dinâmica do sistema tem-se: um referencial $x_{b}, y_{b}, z_{b}$ (ver Figura 2), cuja origem está no centro de massa do corpo do satélite e onde cada um dos três eixos tem a mesma orientação do torque magnético produzido pelos magnetorques. Um segundo referencial $x_{d}$, $y_{d}, z_{d}$, cuja origem está no centro de massa do corpo do satélite. Por fim, é assumido que o corpo rígido é totalmente atuado, ou seja, o conjunto de todos os torques aplicados ao corpo rígido está em um espaço tridimensional euclidiano.

A dinâmica da atitude de um corpo rígido sujeito a um vetor de torque magnético aplicado é dada por (Chaturvedi et al., 2011):

$$
\boldsymbol{J} \dot{\boldsymbol{\omega}}_{b}=\boldsymbol{J} \boldsymbol{\omega}_{b} \times \boldsymbol{\omega}_{b}+\boldsymbol{\tau}
$$

onde $\boldsymbol{J} \in \Re^{3 \times 3}$ é a matriz de inércia do satélite, $\boldsymbol{\omega}_{b} \in \Re^{3}$ é a velocidade angular da referência no corpo do satélite e $\tau \in \Re^{3}$ é o torque aplicado.

Agora, considerando 


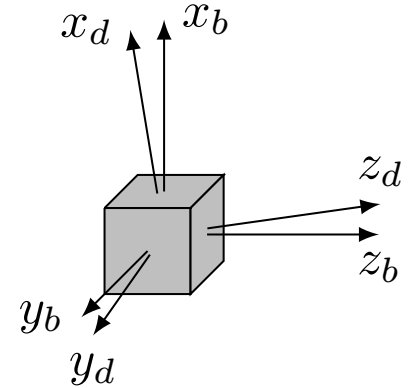

Figura 2. Referenciais do satélite

$$
\breve{\boldsymbol{q}}_{b}=\left[\begin{array}{l}
q_{b 0} \\
\boldsymbol{q}_{b v}
\end{array}\right]=\left[\begin{array}{llll}
q_{b 0} & q_{b 1} & q_{b 2} & q_{b 3}
\end{array}\right]^{T}
$$

o quatérnio unitário $\left(\breve{\boldsymbol{q}}_{b}^{T} \breve{\boldsymbol{q}}_{b}=1\right)$ que representa a atitude do satélite, a cinemática rotacional do corpo do satélite pode ser descrita por:

$$
\begin{aligned}
\dot{q}_{b 0} & =-0.5 \boldsymbol{q}_{b v}^{T} \boldsymbol{\omega}_{b} \\
\dot{\boldsymbol{q}}_{b v} & =0.5\left(q_{b 0} \boldsymbol{\omega}_{b}+\boldsymbol{q}_{b v} \times \boldsymbol{\omega}_{b}\right)
\end{aligned}
$$

Seja

$$
\breve{\boldsymbol{q}}_{d}=\left[\begin{array}{c}
q_{d 0} \\
\boldsymbol{q}_{d v}
\end{array}\right]=\left[\begin{array}{llll}
q_{d 0} & q_{d 1} & q_{d 2} & q_{d 3}
\end{array}\right]^{T}
$$

o quatérnio unitário que representa a atitude desejada para o satélite e

$$
\breve{\boldsymbol{q}}=\left[\begin{array}{l}
q_{0} \\
\boldsymbol{q}_{v}
\end{array}\right]=\left[\begin{array}{llll}
q_{0} & q_{1} & q_{2} & q_{3}
\end{array}\right]^{T}
$$

o quatérnio unitário que representa o erro entre a atitude do satélite e a atitude desejada para o satélite, o qual é obtido por

onde

$$
\breve{\boldsymbol{q}}=\breve{\boldsymbol{q}}_{b}^{*} \otimes \breve{\boldsymbol{q}}_{d}
$$

e

$$
\breve{\boldsymbol{q}}=\breve{\boldsymbol{q}}_{b}^{*} \otimes \breve{\boldsymbol{q}}_{d}=\left[\begin{array}{c}
q_{d 0} q_{b 0}+\boldsymbol{q}_{d v}^{T} \boldsymbol{q}_{b v} \\
q_{d 0} \boldsymbol{q}_{b v}-q_{b 0} \boldsymbol{q}_{d v}-\boldsymbol{q}_{d v} \times \boldsymbol{q}_{b v}
\end{array}\right]
$$

$$
\breve{\boldsymbol{q}}_{b}^{*}=\left[\begin{array}{c}
q_{b 0} \\
-\boldsymbol{q}_{b v}
\end{array}\right]
$$

é o quatérnio conjugado de $\breve{\boldsymbol{q}}_{b}$. A dinâmica e a cinemática do erro entre os quatérnios são expressas por:

$$
\begin{aligned}
\boldsymbol{J} \dot{\boldsymbol{\omega}} & =\boldsymbol{J} \boldsymbol{\omega} \times \boldsymbol{\omega}+\boldsymbol{\tau} \\
\dot{q}_{0} & =-0.5 \boldsymbol{q}_{v}^{T} \boldsymbol{\omega} \\
\dot{\boldsymbol{q}}_{v} & =0.5\left(q_{0} \boldsymbol{\omega}+\boldsymbol{q}_{v} \times \boldsymbol{\omega}\right)=0.5\left(\boldsymbol{Q}_{s} \boldsymbol{\omega}\right)
\end{aligned}
$$

onde

$$
\begin{gathered}
\boldsymbol{q}_{v} \times \boldsymbol{\omega}=\boldsymbol{Q}_{v}^{\times} \boldsymbol{\omega}=\left[\begin{array}{ccc}
0 & -q_{3} & q_{2} \\
q_{3} & 0 & -q_{1} \\
-q_{2} & q_{1} & 0
\end{array}\right] \boldsymbol{\omega}, \\
\boldsymbol{Q}_{s}=q_{0} \boldsymbol{I}+\boldsymbol{Q}_{v}^{\times}, \\
\boldsymbol{Q}_{s}^{*}=q_{0} \boldsymbol{I}-\boldsymbol{Q}_{v}^{\times},
\end{gathered}
$$

e $\boldsymbol{I} \in \Re^{3 \times 3}$ é a matriz identidade,

$$
\boldsymbol{\omega}=\boldsymbol{\omega}_{b}-\boldsymbol{R}(\breve{\boldsymbol{q}}) \boldsymbol{\omega}_{d}
$$

é o erro entre a velocidade angular da referência no corpo do satélite e a velocidade angular desejada $\left(\boldsymbol{\omega}_{d}\right)$ para o corpo do satélite, $\mathrm{e}$

$$
\boldsymbol{R}(\breve{\boldsymbol{q}})=\left(q_{0}^{2}-\boldsymbol{q}_{v}^{T} \boldsymbol{q}_{v}\right) \boldsymbol{I}+2 \boldsymbol{q}_{v} \boldsymbol{q}_{v}^{T}-2 q_{0} \boldsymbol{Q}_{v}^{\times}
$$

é a matriz de rotação entre os referenciais do corpo do satélite e o desejado, a qual faz parte de um grupo ortogonal especial de terceira ordem:

$$
S O(3)=\left\{\boldsymbol{R} \mid \boldsymbol{R} \in \Re^{3 \times 3}, \boldsymbol{R}^{T} \boldsymbol{R}=\boldsymbol{I}, \operatorname{det}(\boldsymbol{R})=1\right\}
$$

\section{SISTEMA INVERSO À ESQUERDA}

Nesta seção, o objetivo é desacoplar a ação entre as componentes do vetor de entrada $\boldsymbol{\tau}$ e as componentes do vetor de saída $\boldsymbol{q}_{v}$, ou seja, cada componente do vetor de entrada agirá apenas sobre uma das componentes do vetor de saída. Para isso, será aplicada a técnica de inversão de sistemas apresentada por Hirschorn (1979), a qual consiste em derivar o vetor de saída $\boldsymbol{q}_{v}$ até que se encontre uma relação com o vetor de entrada do sistema $\boldsymbol{\tau}$, o que, quando aplicado ao sistema da equação (9), resulta em

$$
\begin{aligned}
\ddot{\boldsymbol{q}}_{v} & =0.5\left(\dot{\boldsymbol{Q}}_{s} \boldsymbol{\omega}\right)+0.5\left(\boldsymbol{Q}_{s} \dot{\boldsymbol{\omega}}\right) \\
& =\boldsymbol{C}_{\beta}+\boldsymbol{D}_{\beta} \boldsymbol{\tau}
\end{aligned}
$$

onde

$$
\boldsymbol{C}_{\beta}=0.5\left(\dot{\boldsymbol{Q}}_{s} \boldsymbol{\omega}+\boldsymbol{Q}_{s}\left(\boldsymbol{J}^{-1}(\boldsymbol{J} \boldsymbol{\omega} \times \boldsymbol{\omega})\right)\right)
$$

$\mathrm{e}$

$$
\boldsymbol{D}_{\beta} \boldsymbol{\tau}=0.5\left(\boldsymbol{Q}_{s} \boldsymbol{J}^{-1}\right) \boldsymbol{\tau}
$$

Considerando que se mede todas as variáveis de estado, o sistema inverso para (9) é dado por

$$
\begin{aligned}
\boldsymbol{\tau} & =-\boldsymbol{D}_{\beta}^{-1} \boldsymbol{C}_{\beta}+\boldsymbol{D}_{\beta}^{-1} \boldsymbol{u} \\
& =-\boldsymbol{J} \boldsymbol{Q}_{s}^{-1} \dot{\boldsymbol{Q}}_{s} \boldsymbol{\omega}-\boldsymbol{J} \boldsymbol{\omega} \times \boldsymbol{\omega}+2 \boldsymbol{J} \boldsymbol{Q}_{s}^{-1} \boldsymbol{u}
\end{aligned}
$$

onde $\boldsymbol{u} \in \Re^{3}$ é a entrada para o sistema inverso e

$$
\begin{aligned}
\boldsymbol{Q}_{s}^{-1} & =\boldsymbol{Q}_{s}^{*}+q_{0}^{-1}\left(\boldsymbol{q}_{v} \boldsymbol{q}_{v}^{T}\right) \\
\boldsymbol{Q}_{s}^{-1} \dot{\boldsymbol{Q}}_{s} \boldsymbol{\omega} & =-0.5 q_{0}^{-1}\left(\boldsymbol{\omega}^{T} \boldsymbol{\omega}\right) \boldsymbol{q}_{v}
\end{aligned}
$$

Aplicando como sinal de entrada para o sistema (9) a equação obtida em (17), tem-se que

$$
\ddot{\boldsymbol{q}}_{v}=\boldsymbol{u}
$$

onde é possível perceber que cada elemento do vetor $\boldsymbol{u}$ se relaciona apenas com um elemento do vetor $\boldsymbol{q}_{v}$. Também é possível verificar que a dinâmica entre cada um dos elementos de $\boldsymbol{u}$ e sua respectiva saída é dada por um duplo integrador.

\section{CONTROLADOR PROPOSTO}

Devido o acoplamento do sistema, ao se aplicar um torque em qualquer uma das bobinas, este torque pode influenciar em todas as componentes do quatérnio que representa a atitude do satélite e, desta forma, sendo necessário aplicar torque nas demais bobinas para compensar essa influência. O controlador proposto neste trabalho consiste na inserção de uma função não linear $f\left(\breve{\boldsymbol{q}}, \boldsymbol{\omega}_{b}, \boldsymbol{u}\right)$ entre o sinal de controle tradicional e o sinal de torque que será aplicado ao nanossatélite. Esta função não linear tem como objetivo fazer com que cada torque gerado tenha influência em apenas uma das componentes do vetor $\boldsymbol{q}_{v}$.

O diagrama de blocos simplificado do ADCS do nanossatélite utilizado neste trabalho é detalhado na Figura 3. Nele é possível identificar que o satélite possui dois sensores $\left(S_{\text {sol }}, S_{\text {mag }}\right)$ responsáveis por determinar a atitude do satélite. O erro entre o quatérnio que representa a atitude do corpo do satélite e o quatérnio desejado é obtido a partir da equação (6). O controlador utilizado é um PD para cada um dos erros, os quais irão calcular cada um dos torques.

A correção será realizada por uma expressão oriunda da equação (17), a qual para $q_{0}=0$ não possui solução. A alteração proposta resulta em 


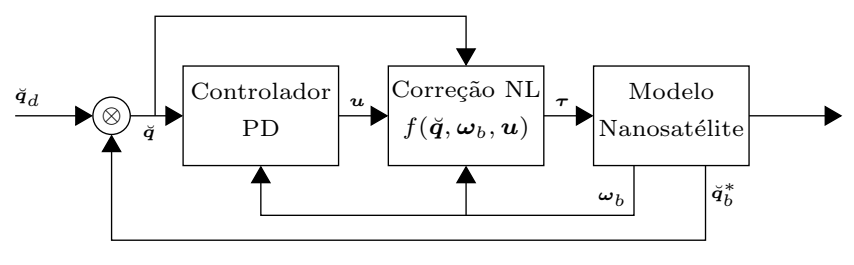

Figura 3. Diagrama de blocos do ADCS proposto

$$
\begin{aligned}
\boldsymbol{\tau}= & f\left(\breve{\boldsymbol{q}}, \boldsymbol{\omega}_{b}, \boldsymbol{u}\right) \\
\boldsymbol{\tau}= & -0.5\left|q_{0}\right| \boldsymbol{J}\left(\boldsymbol{\omega}^{T} \boldsymbol{\omega}\right) \boldsymbol{q}_{v}-\boldsymbol{J} \boldsymbol{\omega} \times \boldsymbol{\omega} \\
& +2 \boldsymbol{J}\left(Q_{s}^{*}+\left(\boldsymbol{q}_{v} \boldsymbol{q}_{v}^{T}\right)\right) \boldsymbol{u}
\end{aligned}
$$

e considerando que $\boldsymbol{\omega}_{d}=0, \boldsymbol{\omega}=\boldsymbol{\omega}_{b}$.

É importante notar que a correção apresentada na equação (20), somente garantirá que cada torque gerado influencia apenas uma componente do vetor $\boldsymbol{q}_{v}$ no caso de $q_{0}=1$. Para os demais casos poderá haver a influência de cada componente de torque gerado em todas as componentes do vetor $\boldsymbol{q}_{v}$ e essa influência está em função de $q_{0}$, sendo que a maior influência ocorrerá quando $q_{0}=0$.

O objetivo do controlador aplicado ao problema de controle de atitude baseado em quatérnios é garantir que $\breve{\boldsymbol{q}}_{b}(t) \rightarrow \breve{\boldsymbol{q}}_{d}(t)$ e $\boldsymbol{\omega}(t) \rightarrow 0$ quando $t \rightarrow \infty$. Desta forma, o rastreio perfeito é conseguido quando

$$
\breve{\boldsymbol{q}}_{b}(t)= \pm \breve{\boldsymbol{q}}_{d}(t) \quad \Leftrightarrow \quad \breve{\boldsymbol{q}}(t)=\left[\begin{array}{c} 
\pm 1 \\
0 \\
0 \\
0
\end{array}\right]
$$

No ADCS tradicional, a lei de controle utilizada é do tipo $\mathrm{PD}$ e pode ser descrita por:

$$
\boldsymbol{u}=-k_{p} \boldsymbol{q}_{v}-k_{d} \boldsymbol{\omega}
$$

onde $k_{p}, k_{d}>0$. Esta lei de controle garante que $\breve{\boldsymbol{q}}(t)=$ $\left[\begin{array}{llll}1 & 0 & 0 & 0\end{array}\right]^{T}$ e $\boldsymbol{w}=\left[\begin{array}{lll}0 & 0 & 0\end{array}\right]$ é um ponto de equilíbrio para o sistema do controle de atitude em malha fechada (ver Figura 3) quando $\boldsymbol{\tau}=\boldsymbol{u}$.

Proposição (Correção não linear) Considere a lei de controle da equação (22) e a correção no sinal de controle da equação (20). Assumindo que se conhece a matriz de inércia $\boldsymbol{J}$, que se mede $\boldsymbol{\omega}, \breve{\boldsymbol{q}}_{b}$ e que $k_{p}, k_{d}>0$, pode-se

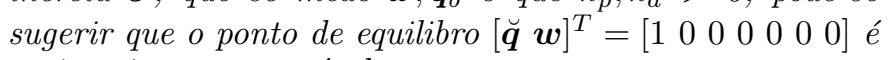
assintoticamente estável.

Nota. Apesar da equação (20) aparentar uma certa complexidade computacional, ela é formada pela repetição de inúmeros termos o que reduz a complexidade computacional.

\section{RESUltados DE SimulaÇÃO}

Por questão de simplicidade e para testar a técnica proposta, todas as simulações possuem duração de $100 \mathrm{~s}$, passo de integração de 0,001 s e matriz de inércia $\boldsymbol{J}=$ $\operatorname{diag}(1,1,1) \mathrm{kgm}^{2}$. O controlador PD utilizou como parâmetros $k_{d}=0,237$ e $k_{p}=0,3$ para o controlador sem a correção. Já para o caso com a correção, das simulações de 01 até 04 , utilizou-se $k_{p}=0,0045$ e para a simulação 05 utilizou-se $k_{p}=0,21$. Os ganhos de ambos os controladores, com ou sem correção, foram escolhidos de tal forma que a saída do sistema apresentasse tempo de estabilização semelhante, exceto no caso da simulação 05 em que a alteração do ganho é para demonstrar que o controlador proposto, se for de interesse do projetista, pode apresentar desempenho melhor que o controlador sem a correção e ainda utilizar menos energia. Por fim é importante destacar que as condições iniciais de cada simulação são apresentadas na Tabela 1, que a norma 2 de um quatérnio unitário qualquer $(\|\breve{q}\|)$ é sempre 1 , $\|\boldsymbol{e}\|=\left\|\left[1-q_{0} ; q_{1} ; q_{2} ; q_{3}\right]^{T}\right\|$ onde $\|\boldsymbol{e}\| \rightarrow 0$ quando $\breve{\boldsymbol{q}}_{b} \rightarrow \breve{\boldsymbol{q}}_{d}$, ou seja, uma forma mais simples de apresentar através de gráfico a evolução dos quatérnios,

$$
\int_{t=0}^{\infty}(\|\boldsymbol{\tau}\| d t)
$$

serve para indicar uma proporção do torque total aplicado pelo controlador e será utilizado para a comparação entre as técnicas de controle.

\begin{tabular}{cccc}
\hline Simul. & $\boldsymbol{\omega}$ & $\boldsymbol{q}_{b v}$ & $\boldsymbol{q}_{d v}$ \\
\hline 01 & {$\left[\begin{array}{c}0,00 \\
0,00 \\
-0,01\end{array}\right]$} & {$\left[\begin{array}{c}0,2 \\
0,4 \\
-0,8\end{array}\right]$} & {$\left[\begin{array}{l}0,0 \\
0,0 \\
0,0\end{array}\right]$} \\
\hline 02 & {$\left[\begin{array}{c}0,07 \\
-0,05 \\
-0,04\end{array}\right]$} & {$\left[\begin{array}{c}-0,5 \\
0,5774 \\
0,4082\end{array}\right]$} & {$\left[\begin{array}{l}0,0 \\
0,0 \\
0,0\end{array}\right]$} \\
\hline \multirow{2}{*}{03} & {$\left[\begin{array}{c}0,30 \\
0,05 \\
-0,12\end{array}\right]$} & {$\left[\begin{array}{c}0,8 \\
-0,2 \\
0,0\end{array}\right]$} & {$\left[\begin{array}{l}0,0 \\
0,0 \\
0,0\end{array}\right]$} \\
\hline 04,05 & {$\left[\begin{array}{l}0,00 \\
0,00 \\
0,00\end{array}\right]$} & {$\left[\begin{array}{c}0,8 \\
-0,2 \\
0,0\end{array}\right]$} & {$\left[\begin{array}{c}-0,4329 \\
0,6645 \\
0,4783\end{array}\right]$} \\
\hline
\end{tabular}

Tabela 1. Condições iniciais de cada simulação

As simulações 01, 02 e 03 são aplicadas para o mesmo quatérnio desejado a partir de diferentes condições iniciais do quatérnio $\breve{\boldsymbol{q}}_{b}$. As simulações buscaram atender a situações distintas como, por exemplo, baixa ou alta velocidade angular inicial. Já as simulações 04 e 05 utilizaram as mesmas condições iniciais e o mesmo quatérnio desejado, o qual é diferente das simulações anteriores. A simulação 04 apresenta uma situação na qual se busca, além de reduzir o consumo energético, um melhor desempenho no transitório do controlador.

Na simulação 01 (Figura 4), é possível observar a evolução ao longo do tempo de todas as componentes do quatérnio $\breve{\boldsymbol{q}}_{b}$ que representa a atitude do corpo do satélite, a velocidade angular $\left(\boldsymbol{\omega}_{b}\right)$ do corpo do satélite e o torque $(\boldsymbol{\tau})$ aplicado ao corpo do satélite para ambos os controladores $\mathrm{PD}$, sem e com a correção não linear. Além disso, é possível verificar as medidas $\|\boldsymbol{e}\|,\|\boldsymbol{\tau}\|$ e $\int(\|\boldsymbol{\tau}\| d t) \times t$ que servem para representar, de forma condensada, a dinâmica do satélite e o torque total aplicado a ele. Baseado no objetivo de apresentar controladores com o mesmo desempenho com relação ao tempo de estabilização, é possível concluir que esse objetivo foi alcançado pois, para ambos os casos, o tempo de estabilização é por volta dos 40 s. Também, a partir do gráfico (i) desta simulação, pode-se comprovar que, para estabilizar o satélite, o controlador com correção não linear apresenta um consumo energético mais eficiente, pois o mesmo utiliza um torque total pelo menos 5 vezes menor que o utilizado pelo controlador PD sem correção. 


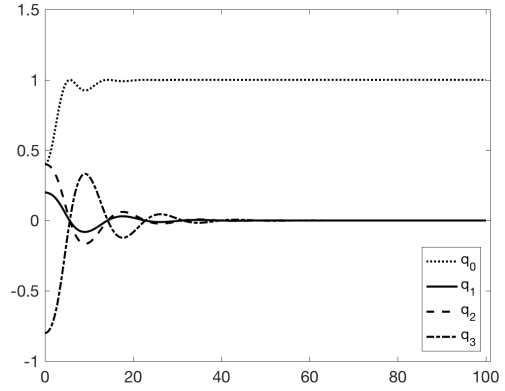

(a) Controlador PD - $\breve{\boldsymbol{q}} \times t(\mathrm{~s})$

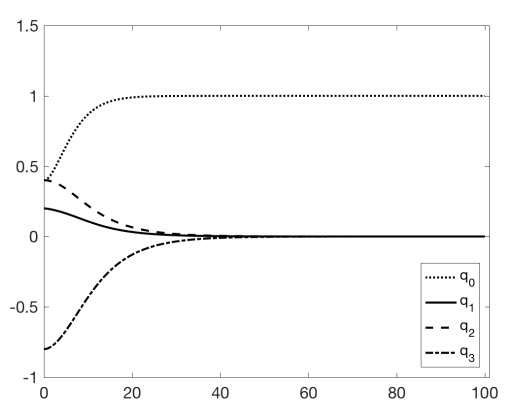

(d) Controlador PD+CNL - $\breve{\boldsymbol{q}} \times t$ (s)

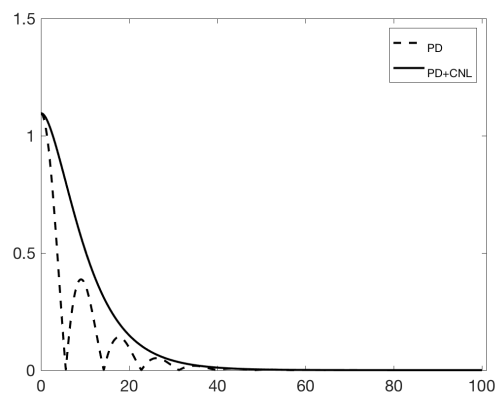

(g) $\|\boldsymbol{e}\| \times t(\mathrm{~s})$

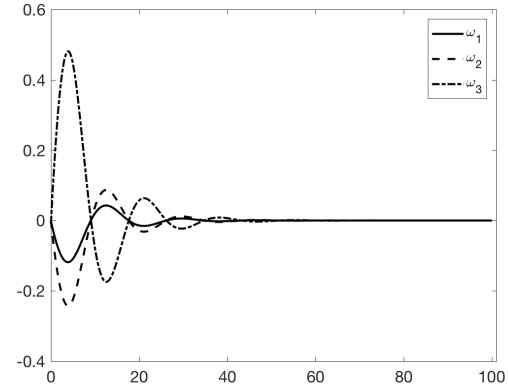

(b) Controlador PD $-\boldsymbol{\omega}(\mathrm{rad} / \mathrm{s}) \times t(\mathrm{~s})$

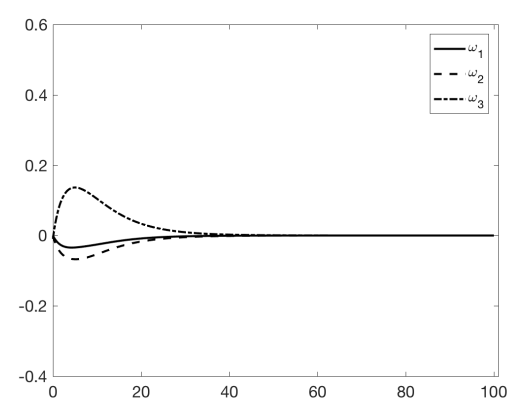

(e) Controlador PD+CNL - $\boldsymbol{\omega}(\mathrm{rad} / \mathrm{s}) \times t(\mathrm{~s})$

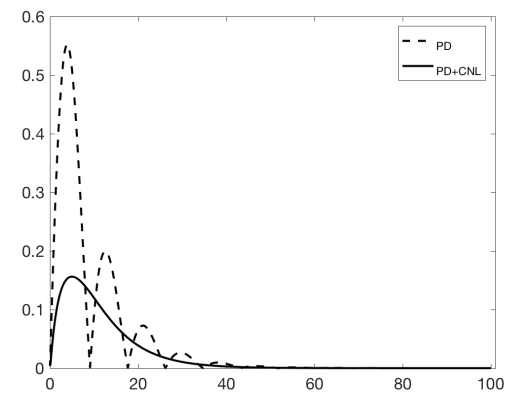

(h) $\|\boldsymbol{\omega}\|(\mathrm{rad} / \mathrm{s}) \times t(\mathrm{~s})$

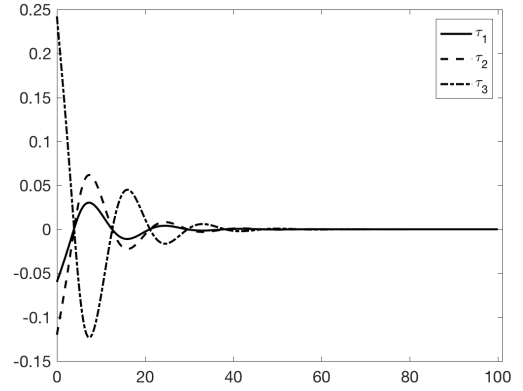

(c) Controlador PD $-\boldsymbol{\tau}(\mathrm{Nm}) \times t(\mathrm{~s})$

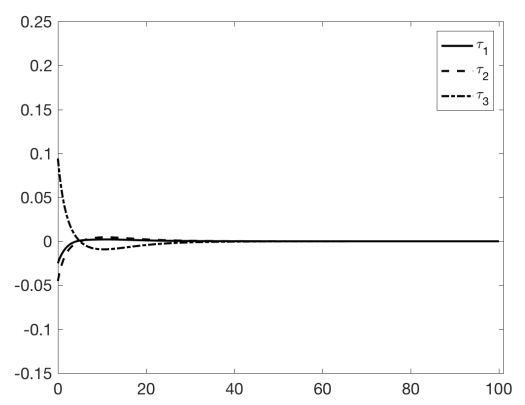

(f) Controlador PD+CNL - $\boldsymbol{\tau}(\mathrm{Nm}) \times t(\mathrm{~s})$

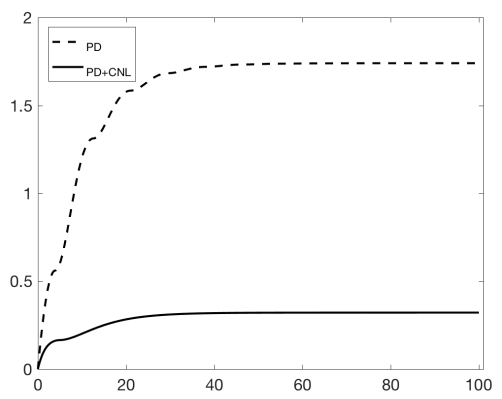

(i) $\int(\|\boldsymbol{\tau}\| d t) \times t(\mathrm{~s})$

Figura 4. Simulação 01 - Baixa velocidade angular inicial com quatérnio desejado na origem

As simulações 02 e 03 (Figuras 5 e 6 , respectivamente) apresentam resultados semelhantes ao da simulação 01, ou seja, mesmo desempenho com relação ao tempo de estabilização e consumo energético mais eficiente por parte do controlador PD com correção não linear.

Com relação à simulação 04 (Figura 7), modifica-se o quatérnio desejado para um valor diferente das simulações anteriores, apenas para demonstrar o funcionamento da técnica para um outro arranjo. De forma semelhante aos casos anteriores, ambos os controladores apresentaram o mesmo tempo de estabilização, sendo que o controlador com correção apresentou uma eficiência energética melhor que o controlador PD sem correção, mais uma vez.

Por fim, a simulação 05 (Figura 8) apresenta um resultado que busca melhorar a eficiência energética e um tempo de estabilização menor para o sistema. Para isso, apenas alterou-se o ganho proporcional do controlador PD com correção não linear. De acordo com a simulação 05, o controlador PD com correção não linear apresentou um tempo de estabilização quase que a metade do caso com o controlador PD sem correção e, ainda, com uma redução de aproximadamente $15 \%$ no torque aplicado.

\section{CONCLUSÕES}

Neste trabalho foi proposta uma alteração do sistema de controle de atitude de um nanossatélite. O controlador PD tradicional do sistema recebeu uma correção não linear, obtida a partir de uma técnica de inversão de sistemas, com o objetivo de reduzir o consumo energético através da redução dos efeitos do acoplamento do sistema. A correção não linear proposta, apesar de aumentar a complexidade dos cálculos, consegue reduzir o consumo, pois a realização dos cálculos extras pelo computador de bordo, equipado com um ARM9, representa um acréscimo insignificante do consumo de energia. Porém, a redução da amplitude dos torques, os quais são gerados por bobinas magnéticas (magnetorques), produzem uma redução do consumo de energia muito superior ao acréscimo pelos novos cálculos. A redução significativa na amplitude dos 


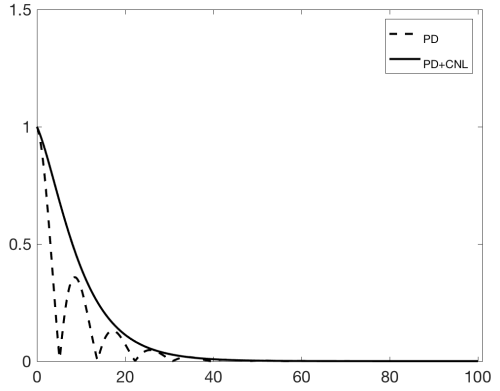

(a) $\|e\| \times t(\mathrm{~s})$

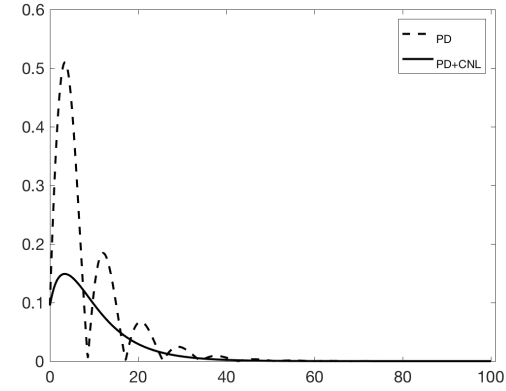

(b) $\|\boldsymbol{\omega}\|(\mathrm{rad} / \mathrm{s}) \times t(\mathrm{~s})$

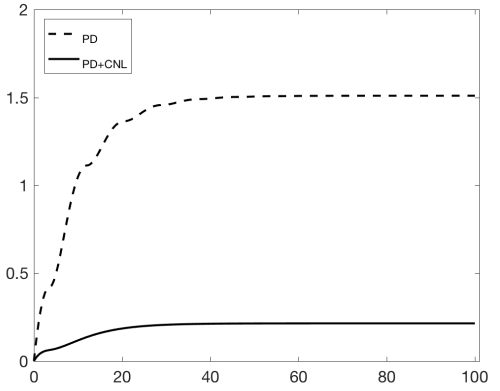

(c) $\int(\|\boldsymbol{\tau}\| d t) \times t(\mathrm{~s})$

Figura 5. Simulação 02 - Velocidade angular inicial em todos os eixos com quatérnio desejado na origem

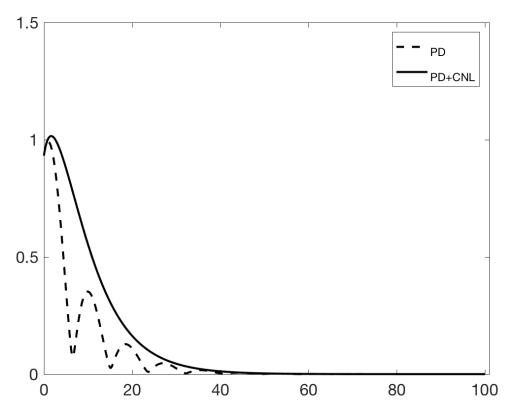

(a) $\|\boldsymbol{e}\| \times t(\mathrm{~s})$

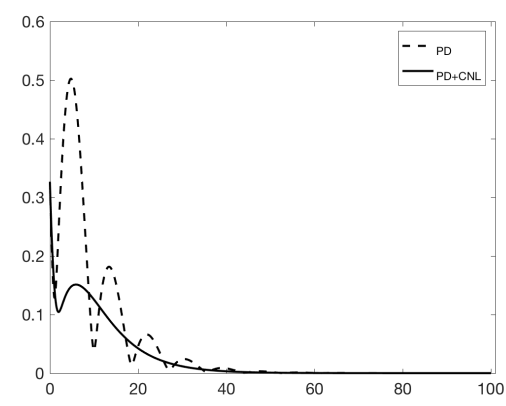

(b) $\|\boldsymbol{\omega}\|(\mathrm{rad} / \mathrm{s}) \times t(\mathrm{~s})$

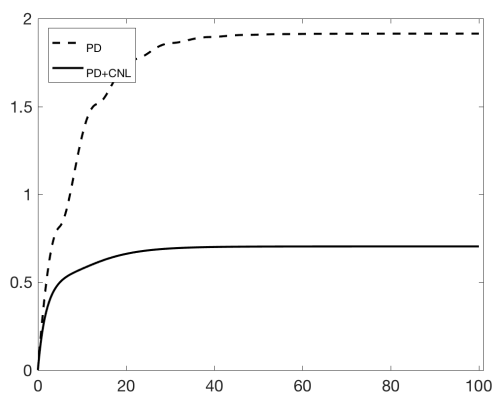

(c) $\int(\|\boldsymbol{\tau}\| d t) \times t(\mathrm{~s})$

Figura 6. Simulação 03 - Velocidade angular inicial maior em dois eixos com quatérnio desejado na origem

torques é observada em todos os resultados de simulação apresentados. Os próximos passos para o desenvolvimento da técnica é a prova de estabilidade, a avaliação com relação à inserção de distúrbios e incertezas no sistema e a utilização dos parâmetros do nanossatélite do CONASAT.

\section{AGRADECIMENTOS}

Os autores agradecem pelo suporte financeiro do INPE/CRN através do projeto CONASAT.

\section{REFERÊNCIAS}

Bang, H., Ha, C.K., and Kim, J.H. (2005). Flexible spacecraft attitude maneuver by application of sliding mode control. Acta Astronautica, 57(11), 841-850.

Chaturvedi, N.A., Sanyal, A.K., and McClamroch, N.H. (2011). Rigid-body attitude control. IEEE Control Systems Magazine, 31(3), 30-51.

Giri, D.K. and Sinha, M. (2019). Robust backstepping magnetic attitude control of satellite subject to unsymmetrical mass properties. Journal of Spacecraft and Rockets, 56(1), 298-305.

Hirschorn, R. (1979). Invertibility of multivariable nonlinear control systems. IEEE Transactions on Automatic Control, 24(6), 855-865.

Ivanov, D., Ovchinnikov, M., and Roldugin, D. (2018). Three-axis attitude determination using magnetorquers. Journal of Guidance, Control, and Dynamics, 41(11), 2455-2462.

Kristiansen, R., Nicklasson, P.J., and Gravdahl, J.T. (2009). Satellite attitude control by quaternion-based backstepping. IEEE Transactions on Control Systems Technology, 17(1), 227-232.

Lai, L.C., Yang, C.C., and Wu, C.J. (2007). Timeoptimal maneuvering control of a rigid spacecraft. Acta Astronautica, 60(10-11), 791-800.

Leeghim, H., Choi, Y., and Bang, H. (2009). Adaptive attitude control of spacecraft using neural networks. Acta Astronautica, 64(7-8), 778-786.

Li, C., Teo, K.L., Li, B., and Ma, G. (2012). A constrained optimal pid-like controller design for spacecraft attitude stabilization. Acta Astronautica, 74, 131 - 140.

Li, Y. and Ye, D. (2019). Robust pid controller for flexible satellite attitude control under angular velocity and control torque constraint. Asian Journal of Control, 118.

Lovera, M., De Marchi, E., and Bittanti, S. (2002). Periodic attitude control techniques for small satellites with magnetic actuators. IEEE Transactions on Control Systems Technology, 10(1), 90-95.

Reis de Mesquita, B.D., Koiti Kuga, H., and Carrara, V. (2017). Estimation and attitude control in conasat's nominal operation mode: An approach for sdre filter and pid control. IEEE Latin America Transactions, 15(5), 835-842.

Shiroma, W.A., Martin, L.K., Akagi, J.M., Akagi, J.T., Wolfe, B.L., Fewell, B.A., and Ohta, A.T. (2011). Cubesats: A bright future for nanosatellites. Central European Journal of Engineering, 1(1), 9-15.

Show, L.L., Juang, J.C., Jan, Y.W., and Lin, C.T. (2003). Quaternion feedback attitude control design: a nonlinear h $\infty$ approach. Asian Journal of Control, 5(3), 406-411. 


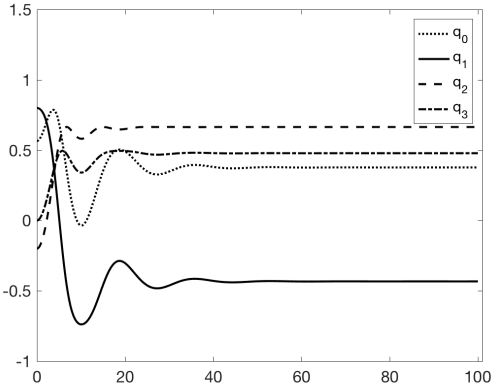

(a) Controlador PD - $\breve{q} \times t($ s)

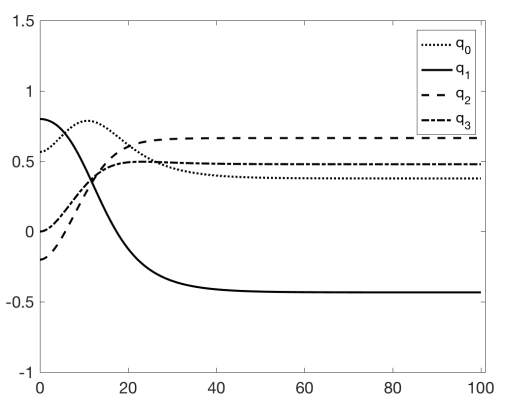

(d) Controlador PD+CNL - $\breve{\boldsymbol{q}} \times t(\mathrm{~s})$

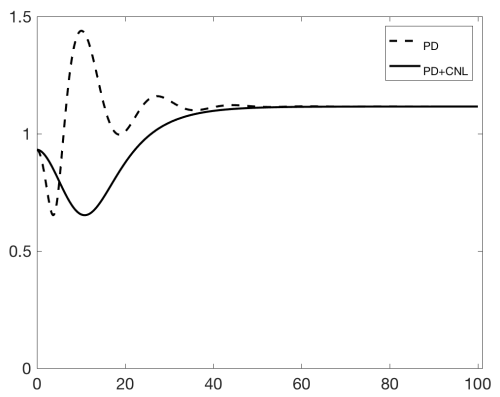

(g) $\|\boldsymbol{e}\| \times t(\mathrm{~s})$

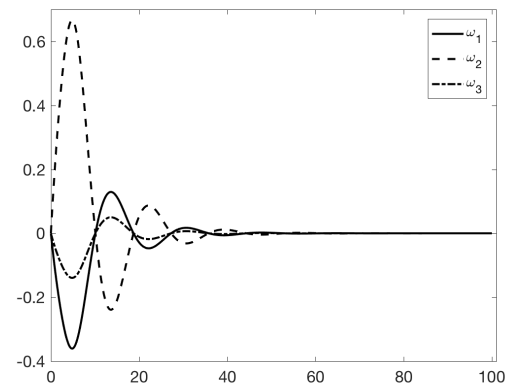

(b) Controlador PD $-\boldsymbol{\omega}(\mathrm{rad} / \mathrm{s}) \times t(\mathrm{~s})$

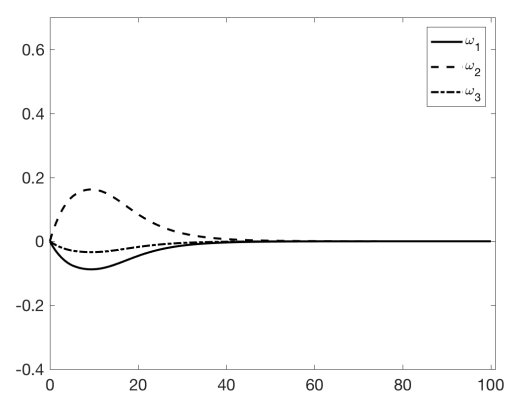

(e) Controlador PD+CNL - $\boldsymbol{\omega}(\mathrm{rad} / \mathrm{s}) \times t(\mathrm{~s})$

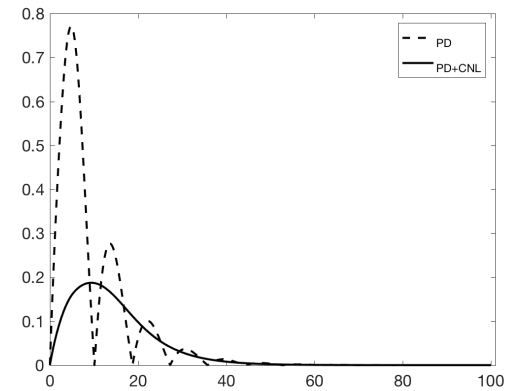

(h) $\|\boldsymbol{\omega}\|(\mathrm{rad} / \mathrm{s}) \times t(\mathrm{~s})$

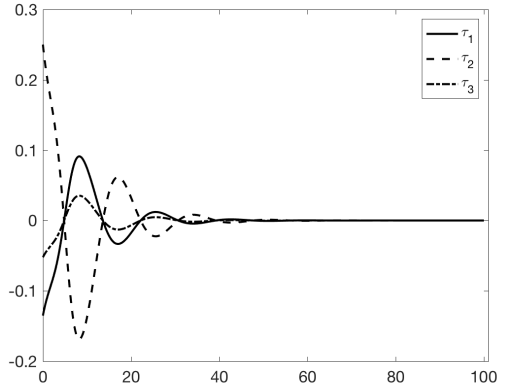

(c) Controlador PD $-\boldsymbol{\tau}(\mathrm{Nm}) \times t(\mathrm{~s})$

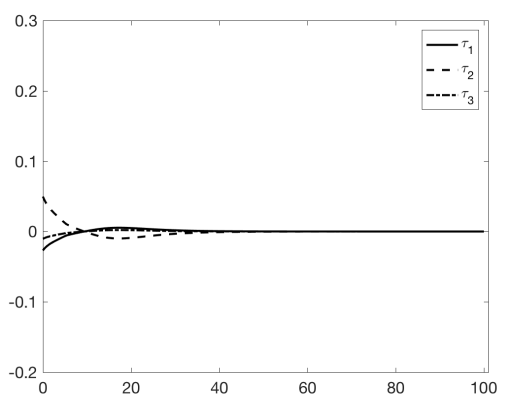

(f) Controlador PD+CNL $-\boldsymbol{\tau}(\mathrm{Nm}) \times t(\mathrm{~s})$

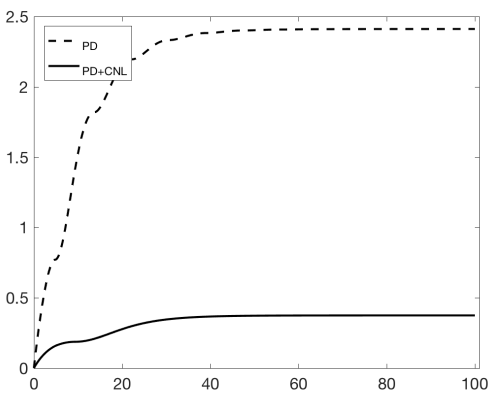

(i) $\int(\|\boldsymbol{\tau}\| d t) \times t(\mathrm{~s})$

Figura 7. Simulação 04 - Velocidade angular inicial nula com quatérnio desejado fora da origem

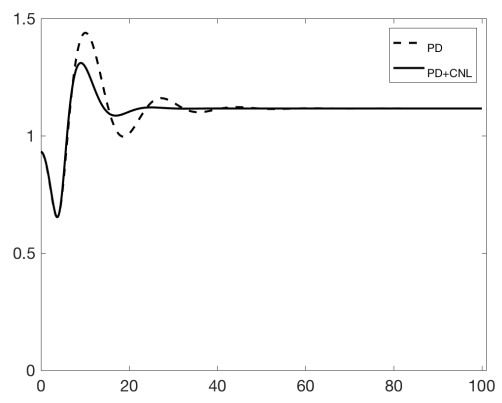

(a) $\|e\| \times t(\mathrm{~s})$

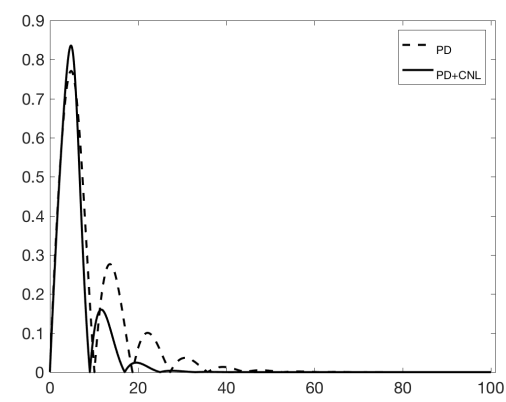

(b) $\|\boldsymbol{\omega}\|(\mathrm{rad} / \mathrm{s}) \times t(\mathrm{~s})$

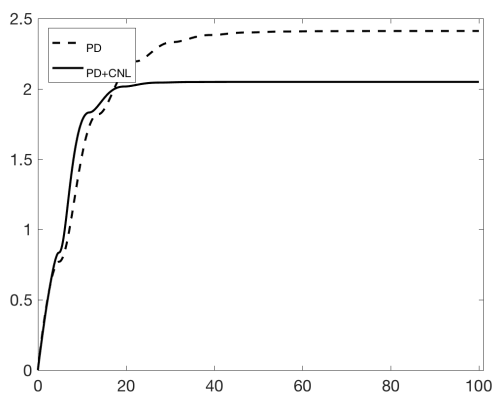

(c) $\int(\|\boldsymbol{\tau}\| d t) \times t(\mathrm{~s})$

Figura 8. Simulação 05 - Velocidade angular inicial nula com quatérnio desejado fora da origem e desempenho transitório mais rápido

Wisniewski, R. (2000). Linear time-varying approach to satellite attitude control using only electromagnetic actuation. Journal of Guidance, Control, and Dynamics, 23(4), 640-647. doi:10.2514/2.4609.
Woellert, K., Ehrenfreund, P., Ricco, A.J., and Hertzfeld, H. (2011). Cubesats: Cost-effective science and technology platforms for emerging and developing nations. Advances in Space Research, 47(4), 663-684. 